\title{
LIMITING DISTRIBUTIONS FOR GEODESICS EXCURSIONS ON THE MODULAR SURFACE
}

\author{
Mark Pollicott
}

University of Warwick

Dedicate to Professor T. Sunada on his 60th birthday

\section{INTRODUCTION}

We begin by recalling a well known result of Dennis Sullivan for geodesic excursions on finite area non-compact surfaces $V$. Let $v(t)$ be position reached after time $t>0$ by a vector traveling at unit speed along the geodesic whose initial unit tangent vector was $v(0)$. This following result describes the behaviour of the distance from $v(t)$ to $v(0)$ for typical geodesics as $t$ tends to infinity.

Theorem 1 (Sullivan) [17,18]. For almost all initial vectors $v(t)$ we have that

$$
\limsup _{t \rightarrow+\infty} \frac{d(v(t), v(0))}{\log t}=1
$$

In the statement of the theorem, the almost all condition is with respect to the Liouville measure, i.e., the natural volume on the unit tangent bundle $T_{1} V$.

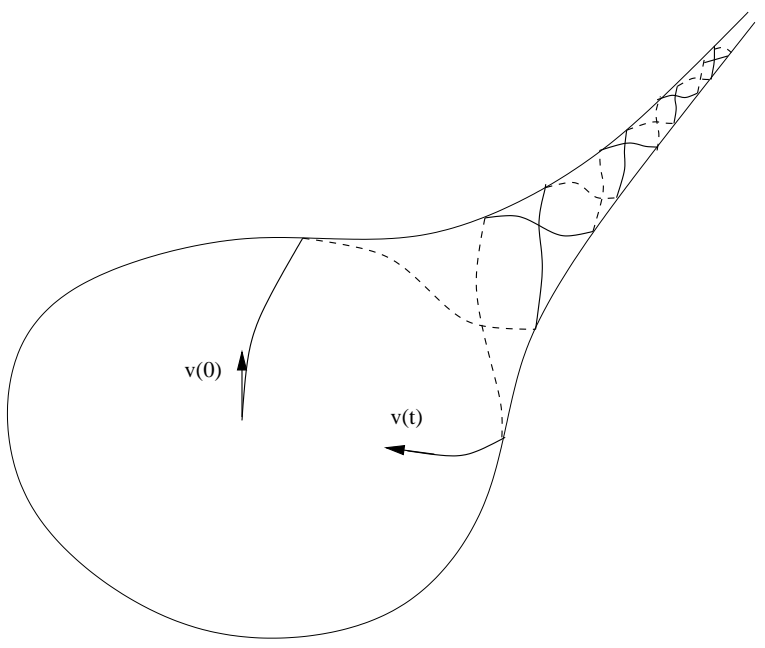

Figure 1. The geodesic $v(t)$ makes an excusion up the cusp 
Theorem 1 is closely related to Khintchine's classical result on diophantine approximation. However, Sullivan's result also extends to more general groups and recently Margulis announced a similar result for horocycles.

The aim of this note is to present the following refinement of Theorem 1 , in the particular case of the Modular surface.

Theorem 2. Let $V$ be the modular surface. For any $y>0$ we have

$$
\begin{aligned}
& \lim _{n \rightarrow+\infty} \mu\left\{v(0) \in T_{1} V: \sup _{0 \leq t \leq T}(d(v(t), v(0)))-\log T \leq \log \left(\frac{6 y}{\pi}\right)\right\} \\
& =\exp \left(-\frac{1}{y}\right) .
\end{aligned}
$$

We first observe that Theorem 2 indeed implies Theorem 1.

Claim. Theorem 2 implies Theorem 1 (in the case of the Modular group).

Proof. The inequality $\lim \sup _{t \rightarrow+\infty} \frac{d(v(t), v(0))}{\log t} \leq 1$ a.e. is the trivial direction, following immediately from the Borel-Cantelli lemma [17, p.231]. The real content of Theorem 1 is the inequality $\limsup _{t \rightarrow+\infty} \frac{d(v(t), v(0))}{\log t} \geq 1$ a.e., which we shall now show can be derived from (0.2). Assume for a contradiction that we can find $\epsilon>0$ such that

$$
\mu\left\{v(0) \in T_{1} V: \limsup _{t \rightarrow+\infty} \frac{d(v(t), v(0))}{\log t} \leq 1-\epsilon\right\} \geq \epsilon .
$$

In particular, we can choose $T_{0}>0$ such that whenever $T \geq T_{0}$ we have that

$$
\begin{aligned}
& \mu\left\{v(0) \in T_{1} V: \sup _{0 \leq t \leq T} d(v(t), v(0)) \leq(1-\epsilon) \log T\right\} \\
& \geq \mu\left\{v(0) \in T_{1} V: \sup _{0 \leq t \leq T} \frac{d(v(t), v(0))}{\log t} \leq 1-\epsilon\right\} \geq \frac{\epsilon}{2} .
\end{aligned}
$$

From (0.2) we see that, given $y>0$ we can choose $T_{1}>0$ such that whenever $T \geq T_{1}$ then

$$
\left|\mu\left\{v(0) \in T_{1} V: \sup _{0 \leq t \leq T} d(v(t), v(0))-\log T<\log \left(\frac{6 y}{\pi}\right)\right\}-e^{-1 / y}\right|<\frac{\epsilon}{4} .
$$

In particular, if we choose , $y>0$ sufficiently small that $e^{-1 / y}<\epsilon / 4$ and choose $T>\max \left\{T_{0}, T_{1}\right\}$ sufficiently large that $0<\epsilon \log T+\log \left(\frac{6 y}{\pi}\right)$, then we have a contradiction.

The original proof of Theorem 1 used a strong variant of the Borel-Cantelli Lemma, which applied to more general groups. However, the proof of Theorem 2 uses quite delicate estimates which do not appear to be available at the same level of generalitiy, although it extends to finite covers of the Modular surface corresponding to Principal Congruence subgroups. This leads to the following natural question.

Problem. For which other Fuchsian groups does there exist an analogue of Theorem 2?

In the first section we describe a distribution result for continued fractions. In the second section we describe the connection between continued fraction expansions and the geodesic flow on the modular surface. In the final section we complete the proof of Theorem 2. 


\section{Distribution of LARgest COEFficients FOR CONTINUED FraCtions}

We first consider a related result due to Galambos for the continued fraction transformation. Let $T:[0,1] \rightarrow[0,1)$ be defined by $T(x)=\frac{1}{x}-\left[\frac{1}{x}\right]$. This preserves the Gauss probability measure $d \mu=\frac{d x}{\log 2(1+x)}$. We can associate to a.e. $(\mu) x \in[0,1]$ its continued fraction expansion $x=\left[a_{0}, a_{1}, a_{2}, \cdots\right]$ where

$$
a_{k}(x)=k \text { if } T^{k} x \in\left[\frac{1}{k+1}, \frac{1}{k}\right) .
$$

This is well defined for $x$ irrational. The following result was proved by János Galambos in 1972 [3].

Proposition 2 (Galambos). For all $y>0$,

$$
\lim _{N \rightarrow+\infty} \mu\left\{x \in[0,1]: \max _{1 \leq n \leq N} a_{n} \leq\left(\frac{y}{\log 2}\right) N\right\}=\exp \left(-\frac{1}{y}\right) .
$$

Subsequently, Philipp observed that there is an error term in this convergence result of the form $O\left(\exp \left(-(\log N)^{\delta}\right)\right)$, for some $\delta>0$ [13].

The following corollary to Proposition 2 is easily deduced (using the same argument as for the claim in the introduction).

Corollary. For a.e. ( $\mu$ ) $x \in[0,1]$ we have that

$$
\limsup _{N \rightarrow+\infty} \frac{\max _{1 \leq n \leq N} \log a_{n}}{\log N}=1 .
$$
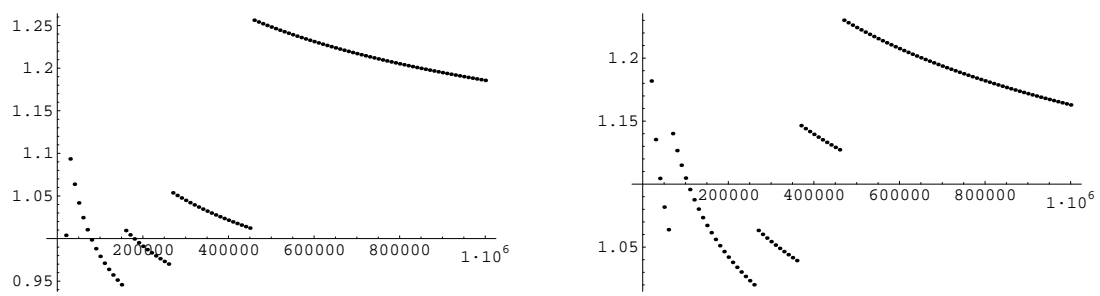

FiguRE 2. Plots of $\frac{\max _{1 \leq n \leq N} \log a_{n}(x)}{\log N}$ for: (i) $x=\pi$; and (ii) $x=\sqrt{\pi}$.

This result can be deduced from the following more general result (using the same argument as in the previous section).

For completeness, we recall the main steps in the proof of Proposition 2, and then discuss its generalizations.

Lemma 1. (The inclusion-exclusion principle) [14]

$$
\begin{aligned}
& \mu\left\{x: \max _{1 \leq k \leq N} a_{n}(x) \leq\left(\frac{y}{\log 2}\right) N\right\} \\
& =1+\sum_{k=1}^{N}(-1)^{k} \sum_{1 \leq i_{1}<\cdots<i_{k} \leq N} \mu\left\{x: \min _{1 \leq j \leq k} a_{i_{j}}(x)<\left(\frac{y}{\log 2}\right) N\right\}
\end{aligned}
$$

We also require some specific estimates. 


\section{Lemma 2.}

(1) For any $n \geq 1$ and $N \geq 1$ we have $\mu\left\{x: a_{n}(x) \geq l\right\}=\frac{\log (1+1 / N)}{\log 2}$

(2) There exists $C>0$ and $0<\theta<1$ such that for $k \geq 2, L \geq 1$ and $i_{1}<\cdots<i_{k}$,

$$
\begin{aligned}
& \left|\mu\left\{x: \min _{1 \leq j \leq k} a_{i_{i}}(x) \geq L\right\}-\prod_{j=1}^{k} \mu\left\{x: a_{i_{j}}(x) \geq L\right\}\right| \\
& \leq \prod_{j=1}^{k-1}\left(1+C \theta^{i_{j+1}-i_{j}}\right) \prod_{j=1}^{k} \mu\left\{x: a_{i_{j}}(x) \geq L\right\}
\end{aligned}
$$

Proof. For the first part, we can first explicitly compute the integral $a_{1}(x)=$ $\int_{0}^{\frac{1}{N}} \frac{d x}{\log 2(1+x)}=\frac{\log (1+1 / N)}{\log 2}$. The case of general $a_{n}(x)$ follows from $T$-invariance of the measure.

The second part follows from exponential mixing of $T$, as formulated in [13] (improving on the classical result of Khintchin [7], [11]), and induction. Alternatively, we can see this by letting $B=[0,1 / N]]$ and considering the operator

$$
\mathcal{L} w(x)=\sum_{n=1}^{\infty} \frac{1}{(x+n)(x+n+1)} w\left(\frac{1}{(x+n)}\right)
$$

We can then write $\mu\left(B \cap T^{-n} B\right)=\int \chi_{B} \mathcal{L}^{n-1}\left(\mathcal{L} \chi_{B}\right) d \mu$. Moreover, the operator $\mathcal{L}$ has maximal eigenvalue 1 , with eigenprojection $\mu(\cdot)$, and a spectral gap when acting on bounded analytic functions on a suitable neighbourhood $D \supset[0,1][9]$. In particular, since $\mathcal{L} \chi_{B}=(L+n)^{-1}$ is analytic we can write $\mu\left(B \cap T^{-n} B\right)=$ $\mu(B)^{2}+O\left(\theta^{n}\right)$. The operator $\mathcal{L} w(x)=\sum_{n=1}^{\infty}(x+n)^{-2} w\left((x+n)^{-1}\right)$. Moreover, the implied constants in the error term are $\mu(B)\left\|\mathcal{L} \chi_{B}\right\|_{\infty}=O\left(\mu(B)^{2}\right)$.

1st estimate: Let $M>0$. Using Lemma 1, we can bound the contribution from terms $M+1 \leq k \leq N$ in (1.1) by:

$$
\begin{aligned}
& \sum_{k=M+1}^{N}(-1)^{k} \sum_{1 \leq i_{1}<\cdots<i_{k} \leq N} \mu\left\{x: \min _{1 \leq j \leq k} a_{i_{j}}(x)<\left(\frac{y}{\log 2}\right) N\right\} \\
& \leq \sum_{k=M+1}^{N}\left(\begin{array}{c}
N \\
k
\end{array}\right)\left(\frac{\log \left(1+\frac{\log 2}{N y}\right)}{\log 2}\right)^{k}(C+1)^{k-1} \leq \sum_{k=M+1}^{N} \frac{1}{k !}\left(\frac{C+1}{y}\right)^{k} .
\end{aligned}
$$

In particular, the Right Hand Side of (1.2) can be made smaller than any given $\epsilon>0$ by choosing $M$ is sufficiently large. 
2nd estimate: Let $m \geq 1$. For each $1 \leq k \leq M$ we can write

$$
\begin{aligned}
& \sum_{\substack{1 \leq i_{1}<\cdots<i_{k} \leq N \\
i_{j+1}-i_{j} \geq m}} \mu\left\{x: \min _{1 \leq j \leq k} a_{i_{j}}(x)<\left(\frac{y}{\log 2}\right) N\right\} \\
= & \sum_{\substack{1 \leq i_{1}<\cdots<i_{k} \leq N \\
i_{j+1}-i_{j} \geq m}}\left(\frac{\log \left(1+\frac{\log 2}{N y}\right)}{\log 2}\right)\left(1+O\left(\theta^{m}\right)\right)^{k-1} \\
= & \left(\begin{array}{c}
N-(m-1)(k-1) \\
k
\end{array}\right) \frac{1}{N^{k}}\left(\frac{1}{y}+O\left(\frac{1}{N}\right)\right)^{k}\left(1+O\left(\theta^{m}\right)\right)^{k} \\
= & \frac{y^{-k}}{k !}(1+O(1 / N))^{k}\left(1+O\left(\theta^{m}\right)\right)^{k-1}
\end{aligned}
$$

3rd estimate: We can bound the contribution of the remaining terms by

$$
\begin{aligned}
& \left(\left(\begin{array}{c}
N \\
k
\end{array}\right)-\left(\begin{array}{c}
N-(m-1)(k-1) \\
k
\end{array}\right)\right)\left(\frac{\log \left(1+\frac{\log 2}{N y}\right)}{\log 2}\right)^{k}(C+1)^{k-1} \\
& =o\left(N^{k}\left(\frac{\log \left(1+\frac{\log 2}{N y}\right)}{\log 2}\right)\right)(C+1)^{k-1}=o\left(\left(1+\frac{1}{N}\right)^{k}\right)(C+1)^{k-1}
\end{aligned}
$$

as $N \rightarrow+\infty$.

Combining these three estimates we see by Lemma 1 that

$$
\begin{aligned}
& \mu\left\{x: \max _{1 \leq k \leq N} a_{n}(x) \leq\left(\frac{y}{\log 2}\right) N\right\} \\
& =1+\sum_{k=1}^{M}(-1)^{k} \frac{y^{-k}}{k !}\left(1+O\left(\frac{1}{N}\right)\right)^{k}\left(1+O\left(\theta^{m}\right)\right)^{k-1} \\
& +o\left(\left(1+\frac{1}{N}\right)^{k-1}(C+1)^{k}\right)+O(\epsilon)
\end{aligned}
$$

Letting first $N \rightarrow \infty$, then $m \rightarrow+\infty$ and then finally $M \rightarrow \infty$, the Right Hand Side of this identity converges to $e^{-\frac{1}{y}}$, as required. This completes the proof of Proposition 2.

Remarks.

(1) Guivarc'h and Le Jan showed that for a.e.( $\mu) 0 \leq x \leq 1$, we have that the distribution of $\frac{1}{n} \sum_{k=1}^{n}(-1)^{k} \log a_{n}$ converges to a Cauchy distribution [4].

(2) Proposition 2 also has a simple interpretation in terms of the how quickly typical $T$-orbits approach zero (and as such can be compared with Collet's result on Gumbel's law [2]), i.e.,

$$
\lim _{N \rightarrow+\infty} \mu\left\{x: \max _{1 \leq n \leq N}-\log \left|T^{n}(x)\right|-\log N \leq \log \left(\frac{y}{\log 2}\right)\right\}=\exp \left(-\frac{1}{y}\right)
$$


(3) Recall that for $n \geq 1$ the rational number given by the finite continued fraction expansion

$$
\frac{p_{n}(x)}{q_{n}(x)}=\left[a_{1}(x), \cdots, a_{n}(x)\right]
$$

is called the $n$th convergent to $x$. The following is a natural generalization of Proposition 2: For any $m \geq 2,1 \leq p, q, \leq m$ with greatest common divisor 1 and $y>0$ we have

$$
\lim _{N \rightarrow+\infty} \mu\left\{x \in[0,1]: \max _{\substack{1 \leq n \leq N \\ p_{n}(x)=p(\bmod m) \\ q_{n}(x)=q(\bmod m)}} a_{n}(x) \leq\left(\frac{y}{\log 2}\right) N\right\}=\exp \left(-\frac{J(m)}{y}\right) .
$$

Where $J(m)=m^{2} \prod_{p \mid m}\left(1-p^{-2}\right)$. Lemma 2 (1) can be replaced by: For any $n \geq 1$ we have

$$
\mu\left\{x: a_{n}(x) \geq l,\left(\begin{array}{c}
p_{n}(x) \\
q_{n}(x)
\end{array}\right)=\left(\begin{array}{c}
p \\
q
\end{array}\right)(\bmod m)\right\}=\frac{1}{J(m)} \frac{\log (1+1 / l)}{\log 2} .
$$

We also need to replace Lemma $2(2)$ by a corresponding mixing condition for the skew product $\widehat{T}:[0,1] \times G(2, \mathbb{Z}, / m \mathbb{Z}) \rightarrow[0,1] \times G(2, \mathbb{Z}, / m \mathbb{Z})$ defined by

$$
\widehat{T}(x, g)=(T(x), g g(x)) \text { where } g(x)=\left(\begin{array}{cc}
0 & 1 \\
1 & a_{0}(x)
\end{array}\right) \text {. }
$$

For $n \geq 2$, we have $g(x) g(T x) \cdots g\left(T^{n-1} x\right)=\left(\begin{array}{cc}p_{n-1} & p_{n} \\ q_{n-1} & q_{n}(x)(x)\end{array}\right)$. Let $\widehat{\mu}=\mu \times \lambda$ where $\lambda$ is the Haar measure on $G$ then, as in Lemma 2(2), there exists $0<\theta<1$ such that if $B=\left\{x: a_{1}(x) \geq l\right\} \times\{g\}$ then

$$
\int \chi_{B} \chi_{B} \circ \widehat{T}^{n} d \widehat{\mu}=\widehat{\mu}(B)^{2}\left(1+O\left(\theta^{n}\right)\right)
$$

The analogue of Lemma 2.2 holds, and (1.5) follows by analogy with the proof of Proposition 2.

\section{SymboliC DYNAMICS FOR GEODESIC FLOWS}

In this section we recall the connection between the continued fraction transformation $T:[0,1] \rightarrow[0,1]$ and the geodesic flow on the Modular surface. These are described in detail in the articles [1], [16], , [15], [9], [4].

We recall that the Modular surface $V$ is a finite area non-compact surface of constant curvature $\kappa=-1$ (actually, with two ramification points and a cusp). We recall the definition of the geodesic flow. The surface $V$ has area $2 \pi / 3$ and thus the unit tangent bundle $T_{1} V$ has volume $4 \pi^{2} / 3$.

Definition. Let $\phi_{t}: T_{1} V \rightarrow T_{1} V$ be the geodesic flow (i.e., given $v=v(0) \in T_{1} V$ we choose a geodesic $\gamma: \mathbb{R} \rightarrow V$ which at time $t=0$ has derivative $\dot{\gamma}(0)=v$ and then set $\left.\phi_{t} v=v(t)=\dot{\gamma}(0)\right)$. 
The universal cover of $V$ is a the upper half-plane $\mathbb{H}^{2}=\{x+i y: y>0\}$. The lift of the metric on $V$ to $\mathbb{H}^{2}$ is the usual Poincaré metric $d s^{2}=\left(d x^{2}+d y^{2}\right) / y^{2}$. The covering group corresponds to $\Gamma=P S L(2, \mathbb{Z})$, acting by linear fractional transformations, and we can write $V=\mathbb{H}^{2} / \Gamma$. In particular, the generators are $T: z \mapsto z+1$ and $S: z \mapsto-1 / z$. We can choose a fundamental domain for $\Gamma$ of the form

$$
F=\left\{z \in \mathbb{H}^{2}:-\frac{1}{2} \leq \operatorname{Re}(z) \leq \frac{1}{2} \text { and }|z| \geq 1\right\}
$$

whose boundaries are identified using $S$ and $T$.

The lift of a typical geodesic $\gamma$ on $V$ is a geodesic $\tilde{\gamma}$ on $\mathbb{H}^{2}$, typically corresponding to circular arc with distinct end points $\tilde{\gamma}(-\infty), \tilde{\gamma}(+\infty) \in \mathbb{R}$ (or, exceptionally, a vertical line). Assuming that $\gamma(-\infty)<\gamma(+\infty)$ we can choose a particular lift so that $-1 \leq \tilde{\gamma}(-\infty) \leq 0$ and $\tilde{\gamma}(+\infty) \geq 1$. On the other hand, if $\gamma(+\infty)<\gamma(-\infty)$ we can choose a particular lift so that $-1 \leq \tilde{\gamma}(+\infty) \leq 0$ and $\tilde{\gamma}(-\infty) \geq 1$.

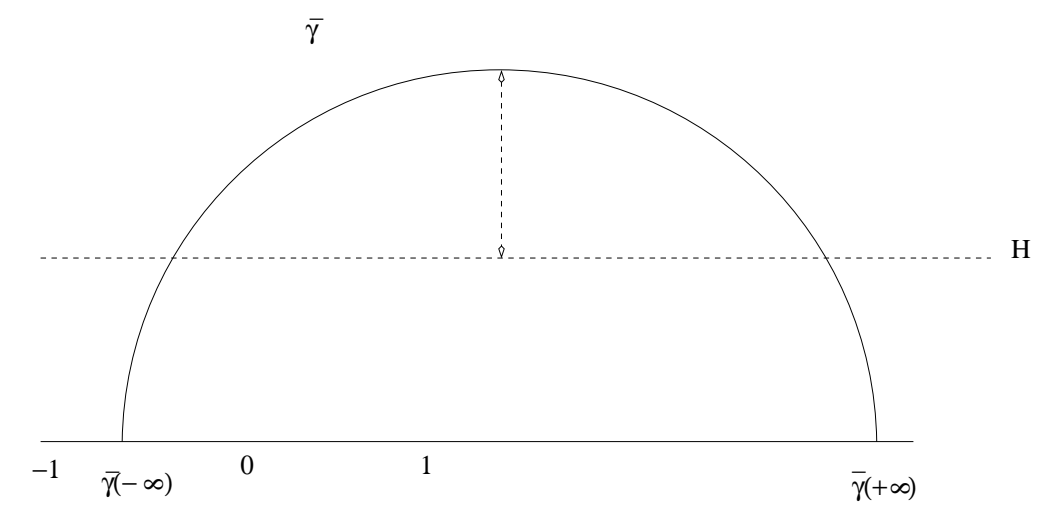

FigurE 3. The lift of a geodesic from the Modular surface to the upper half plane

In either case, we can associate to the geodesic a pair of points $(x, y) \in[0,1]^{2}$, where $\{-y, 1 / x\}=\{\gamma(-\infty), \gamma(+\infty)\}$. We label these two cases by an index $\epsilon \epsilon$ $\mathbb{Z} / 2 \mathbb{Z}$.

There is a natural symbolic dynamics that relates the geodesic flow on the modular surface to a suspension flow over the natural extension of continued fraction map (with a $\mathbb{Z} / 2 \mathbb{Z}$-extension). More precisely, we define

$$
\bar{T}:[0,1]^{2} \times \mathbb{Z} / 2 \mathbb{Z} \rightarrow[0,1]^{2} \times \mathbb{Z} / 2 \mathbb{Z}
$$

almost everywhere by

$$
\bar{T}(x, y ; \epsilon)=\left(T(x), \frac{1}{\left[\frac{1}{x}\right]+y} ; 1-\epsilon\right)
$$

We can define a suspension function $r:[0,1]^{2} \rightarrow[0,1]^{2}$ by $r(x, y)=-2 \log x$, and then define a space

$$
\Lambda=\{(x, y, u) \in[0,1] \times[0,1] \times \mathbb{R}: 0 \leq u \leq r(x)\}
$$


where we identify $(x, y, \epsilon ; r(x))=(\bar{T}(x, y), 1-\epsilon ; 0)$. Finally, we define the suspended flow $\psi_{t}: \Lambda \rightarrow \Lambda$ locally by $\psi_{t}(x, y, \epsilon ; u)=(x, y, \epsilon ; u+t)$, subject to the identifications.

Let $\nu$ denote the Liouville measure on $T_{1} V$ (i.e., normalized volume on $T_{1} V$ ) then $\nu$ is a $\phi$-invariant probability measure. There is a natural isomorphism between the geodesic flow and the suspended flow. Furthermore, the measure $\nu$ projects to the natural extension of the Gauss measure on $[0,1]^{2}$. Of course, the suspension function $r$ can be chosen up to the addition of a coboundary. However, this property of the measure $\nu$ identifies the specific choice $r(x, y)=2 \log x$ as being in the cohomology class (using simple ideas from thermodynamic formalism, e.g., the periodic points $T^{n} x=x$ must satisfy $\log \left(T^{n}\right)^{\prime}(x)=\sum_{i=0}^{n-1} r\left(T^{i} x\right)$ and then Livsic's Theorem applies).

The coding of geodesics on suitable finite covers of the Modular surface is described in [10]

\section{Proof of Theorem 2.}

Consider a geodesic $\bar{\gamma}$ on $\mathbb{H}^{2}$ with end points $\bar{\gamma}(-\infty)$ and $\gamma(+\infty)$, as described in the previous section. We can interpret the height of its excursion into the cusp to be the distance of its highest point from the horizontal line $H=\{z: \operatorname{Im}(z)=1\}$. If the geodesic corresponds to a pair $(x, y) \in[0,1]^{2}$ then we say that the points $\bar{T}^{n}(x, y) \in[0,1]^{2}, n \geq 0$, correspond to the geodesics $n$th excustion into the cusp.

To make use of the symbolic dynamics in the previous section, we need the following estimates specific to the present problem.

Lemma 3. For almost all geodesics,

(1) the excursion into the cusp of the geodesic $\bar{\gamma}$ corresponding to $(x, y) \in[0,1]^{2}$ is to a height $\log \frac{1}{2}(y+1 / x)-1$; and

(2) the (non-euclidean) height of the nth excursion is asymptotic to $\log a_{n}(x)$ and the time of the nth excursion is asymptotic to $\frac{\pi}{6 \log 2} n$.

Proof. The basic framework was described in [10]. Let us assume that $(x, y) \in[0,1]^{2}$ gives rise to a geodesic $\bar{\gamma}$ with end points $\bar{\gamma}(-\infty)=x$ and $\bar{\gamma}(+\infty)=1 / y$, say. Then the geodesic is a semicircular arc centred on the midpoint $\frac{1}{2}\left(-x+\frac{1}{y}\right)$ of $-y$ and $\frac{1}{x}$, and whose Euclidean height above the real line is $\frac{1}{2}\left(y+\frac{1}{x}\right)$. In particular, the hyperbolic distance of the highest point from $H$ is

$$
\int_{1}^{\frac{1}{2}\left(y+\frac{1}{x}\right)} \frac{d \xi}{\xi}=\log \left(\frac{1}{2}\left(y+\frac{1}{x}\right)\right) .
$$

This completes the proof of part (1).

Let $\left(x_{n}, y_{n}\right)=\bar{T}^{n}(x, y)$, then by the first part the height of the $n$th geodesic excursion is $\log \frac{1}{2}\left(y_{n}+1 / x_{n}\right)$. However, since $a_{n}(x) \leq y_{n}+1 / x_{n} \leq a_{n}(x)+2$ we see that this term is asymptotic to $\log a_{n}(x)$. Furthermore, the time to the $n$th excursion is estimated by the sum $r(x, y)+r(\bar{T}(x, y))+\cdots+r\left(\bar{T}^{n-1}(x, y)\right)$. In addition, since we have maked the choice $r(x, y)=-2 \log x$ the Birkhoff ergodic theorem shows that for almost all points this is asymptotic to $\left(-2 \int_{0}^{1} \frac{\log x}{\log 2(1+x)} d x\right) n=\frac{\pi}{6 \log 2} n$, for a.a. $(\mu) x$. 
In particular, for almost all geodesics the successive local maxima for the function $\exp (d(v(t), v(0)))$ will be asymptotic to the successive values $a_{n}(x)$. Finally, using Proposition 2, we have that

$$
\begin{aligned}
& \lim _{n \rightarrow+\infty} \mu\left\{v \in T_{1} V: \max _{1 \leq t \leq T} \exp (d(v(t), v(0))) \leq\left(\frac{y}{\log 2}\right) \frac{6 \log 2}{\pi} T\right\} \\
& \lim _{n \rightarrow+\infty} \mu\left\{x \in[0,1]: \max _{1 \leq k \leq N} a_{n} \leq\left(\frac{y}{\log 2}\right) N\right\}=\exp \left(-\frac{1}{y}\right)
\end{aligned}
$$

This completes the proof of Theorem 2 .

\section{REFERENCES}

1. R. Adler and L. Flatto, Geodesic flows, interval maps, and symbolic dynamics, Bull. Amer. Math. Soc. 25 (1991), 229334.

2. P. Collet, Statistics of closed return for some non-uniformly hyperbolic systems, Ergodic Theory and Dynamical Systems 21 (2001), 401-420.

3. J. Galambos, The distribution of the largest coefficient in the continued fraction expansions, Quart. J. Math. 23 (1972), 147-151.

4. Y. Guivarc'h and Y. Le Jan, Asymptotic winding of the geodesic flow on modular surfaces and continuous fractions, Ann. Sci. E.N.S. 26 (1993), 23-50.

5. H. Jager and P. Lairdet, Distribution arithmetqiues des denominateurs de convergents de fractions continues 91 (1988), 181-197.

6. S. Katok and I. Ugacovici, Symbolic dynamics for the Molular surface and beyond, Bull. Amer. Math. Soc. 44 (2007), 97-132.

7. A. Khintchin, Continued fractions, Dover, Mineola.

8. C. Kraaikamp and A. Lopes, The theta group and the continued fraction expansion with even partial quotients, Geom. Dedicata 59 (1996), 293-333.

9. D. Mayer, The thermodynamic formalism approach to Selberg's zeta function for $P S L(2, \mathbb{Z})$, Bull. Amer. Math. Soc. 25 (1991), 55-60.

10. D. Moeckel, Geodesics on modular surfaces and continued fractions, Ergod. Th. and Dynam. Sys. 2 (1982), 69-82.

11. W. Philipp, Some metrical theorems in number theory, Pacific J. Math. 20 (1967), 109-126.

12. W. Philipp, Some metrical theorems in number theory II, Duke Math. J. 38 (1970), 447-458.

13. W. Philipp, A conjecture of Erdös on continued fractions, Acta Aithmetica 28 (1976), 379386.

14. A. Renyi, Foundations of probability, Holden Day, San Francisco, 1970.

15. C. Series, Symbolic dynamics for geodesic flows, Acta Math. 146 (1981), 103128.

16. C. Series, The modular surface and continued fractions, J. London Math. Soc 31 (1985), 601-625.

17. D. Sullivan, Disjoint spheres, approximation by imaginary quadratic numbers, and the logarithm law for geodesics, Acta Mathematica 149 (1982), 215-237.

18. D. Sullivan, Discrete conformal groups and measurable dynamics, Bull. Amer. Math. Soc. 6 (1982), 57-73.

Department of Mathematics, Warwick University, Coventry, CV4 7AL, UK 